\title{
Qual o significado do trabalho para as pessoas com e sem deficiência física?1
}

\author{
Camila de Sousa Pereira - Universidade Federal de São Carlos² \\ Almir Del Prette - Universidade Federal de São Carlos \\ Zilda Aparecida Pereira Del Prette - Universidade Federal de São Carlos
}

\begin{abstract}
Resumo
Este estudo classificou aspectos positivos e negativos associados ao trabalho por 27 trabalhadores com deficiência física (TDF) em comparação com outros 27 sem deficiência (TND). Os grupos foram emparelhados em sexo, idade, estado civil, nível econômico e função. Os instrumentos utilizados foram: Critério de Classificação Econômica Brasil e Questionário sobre Aspectos do Trabalho. As respostas foram analisadas qualitativa e quantitativamente. Os resultados identificaram seis classes de aspectos positivos (cidadania, conciliação de interesses, finanças, relações sociais, valorização pessoal e valorização profissional) e cinco de negativos (desgaste físico e emocional, desvalorização profissional, dificuldades interpessoais, jornada de trabalho extensa e mecanização do trabalho). A análise estatística apresentou diferença somente na classe jornada de trabalho extensa, com freqüência maior para TND. Conclui-se que a deficiência física não foi fator determinante na valorização de aspectos positivos e negativos do trabalho. Discute-se a importância do trabalho para o desenvolvimento pessoal e profissional das pessoas com deficiência.

Palavras-chave: Educação especial; Relações de trabalho; Deficiência física.
\end{abstract}

\section{What meaning of work for the people with and without physical disability?}

\begin{abstract}
This study classified positive and negative job related aspects for 27 workers with physical disability (TDF) in comparison with 27 others without disability (TND). The groups were paired in sex, age, marital status, economic level and function. The tools used were: Criterion of Brazil Economic Classification and Questionnaire about Aspects of the Work. The answers were analyzed by qualitative and quantitative methods. the results identified six classes of positive aspects (citizenship, combination of interest, finances, personal fulfillment, professional recognition, social relations) and five negative aspects (difficulty in interpersonal relations, mechanical work, physical and emotional stress, professional non recognition, extensive shift work). The statistical analysis presented difference only in the class extensive shift work, with bigger frequency for TND. Concludes that the physical disability was not a significant factor in the valorization of positive and negative aspects provided by the job. The importance of this work for the personal and professional development of people with disability is discussed. Keywords: Special education; Workplace relations; Physical disability.
\end{abstract}

\section{Introdução}

O significado do termo trabalho apresenta-se sob diferentes concepções, ao longo da história, influenciado pelas idéias e pelas modificações da sociedade. O conceito predominante, extraído de Bottomore (1988) e também de Braverman (1987), é que o trabalho constitui-se em atividade exclusivamente humana direcionada para uma finalidade. O que se denomina por trabalho compõe-se de um conjunto de ações com um significado próprio de transformação do homem e da natureza. A predominância da forma de pensar o trabalho depende das condições sócio-históricas em que a pessoa está circunscrita, dos recursos disponíveis, da posição social, das condições de trabalho, entre outros fatores pessoais, conjunturais e estruturais (Borges \& Yamamoto, 2004). Nessa direção, porém sob uma perspectiva psicológica, Borges e AlvesFilho (2001) esclarecem que o significado do trabalho pode ser compreendido como uma cognição subjetiva capaz de refletir [o] e de se fazer refletir [no] momento histórico da sociedade.

O trabalho, como uma atividade quase-livre, voltado a suprir necessidades imediatas do homem, praticamente desapareceu, ocorrendo uma apropriação e uma organização econômico-cultural da força do trabalho. Esse processo engendrou conflitos reduzidos à concepção trabalho versus capital. Nesse embate, que pode ser representado pelo movimento de um pêndulo, ganhos da

\footnotetext{
${ }^{1}$ Apoio Capes.

2 Endereço para correspondência:

Centro de Educação e Ciências Humanas - Departamento de Psicologia - Laboratório de Interação Social/LIS

Rodovia Washington Luis - km 235 - Caixa Postal, 676 - 13565-905 - São Carlos-SP

Tel.: (16) 3351-8447 - 3351-8361

E-mail: cspereira@iris.ufscar.br
} 
força do trabalho eram seguidos de conquistas da classe trabalhadora. $\mathrm{Na}$ atualidade, os ganhos do capital, revestidos de terminologias ratificadas, como neoliberalismo e globalização, geraram desregulamentação progressiva no mercado, instituindo novos padrões organizacionais e tecnológicos e mudanças nas relações sociais de produção. Antunes (2003) argumenta que o aumento da produtividade, com as novas formas de organização e gestão do trabalho, pode ser analisado pela ampliação da exploração e pelo rígido controle sobre a produção. Diminuição de renda, pressão por produtividade, precarização das condições de trabalho, flexibilidade, terceirização das tarefas, são algumas das características presentes no cenário econômico de muitos países (Antunes, 2003; Chahad, 2003; Forrester, 1997; Kovács, 2004). Em decorrência dessas transformações, vasta literatura revela o impacto da organização do trabalho sobre a saúde dos trabalhadores (Dejours, Abdoucheli \& Jayet, 1994; Mendes, Borges \& Ferreira, 2002). Como exemplo, Glina e Rocha (2003) analisaram as implicações da atividade profissional de 67 estagiários e cinco supervisores do setor de cobrança de um banco e obtiveram como resultados mais freqüentes: inabilidade para "desligar-se" mentalmente do trabalho e relaxar, fadiga, irritabilidade, tensão, desânimo e insônia. Além disso, 46,3\% dos participantes apresentaram a clássica relação entre sintomas de estresse e dor muscular.

A noção cognitiva do significado do trabalho supõe que o valor trabalho é introjetado no processo educativo. Todavia, considerando as condições atuais sobre o trabalhador, os que estão no mercado de produção experimentam sentimentos contraditórios entre o bemestar por fazer parte do contingente dos inseridos e a angústia pela permanente ameaça de descartamento. Por outro lado, os sobrantes, além dos problemas financeiros, podem se perceber "inferiorizados" pela exclusão. A contribuição positiva da inserção pode ser observada em estudos que revelam esses aspectos favoráveis associados à ocupação profissional (Chadsey \& Beyer, 2001; Ragazzi, 2001; Sarriera, Câmara \& Berlim, 2000; Sarriera, Silva, Kabbas \& Lópes, 2001; Vash, 1988).

No estudo de Sarriera e colaboradores (2001), a investigação a respeito da construção da identidade profissional de 36 jovens mostrou que a inserção laboral representa uma perspectiva de transformação da realidade de exclusão social, por uma melhoria do nível de qualidade de vida. Buscando comparar os significados do trabalho entre um grupo de empregados e outro de desempregados, Sarriera e colaboradores (2000) verificaram que o grupo de desempregados apresentou menor sentimento de tranqüilidade, bem como de segurança pessoal, de autorealização, de valorização, confiança, gratidão e motivação quando comparado ao grupo de empregados. Além desses aspectos, o grupo de desempregados apresentou maior passividade, maior valor atribuído ao dinheiro e menor valor atribuído à amizade.

$\mathrm{Na}$ área da Educação Especial, a relevância do trabalho para a construção da identidade, o exercício da cidadania e o desenvolvimento socioemocional das pessoas com deficiência é também defendida por uma extensa literatura nacional e internacional (Amaral, 1994; Anache, 1996; Antonello, 1996; Araújo, Escobal \& Goyos, 2006; Carreira, 1997; Chadsey \& Beyer, 2001; Dalferth, Schnappauf \& Sommerer, 1995; Escobal, Araújo \& Goyos, 2005; Galvani, 2001; Jaime \& Carmo, 2005; Lancillotti, 2003; Manzini, 1989; Ragazzi, 2001; Tanaka \& Manzini, 2005; Vash, 1988). Segundo Vash (1988), por meio do trabalho, as pessoas adquirem recompensas externas (poder, salário, prestígio) e internas (autorealização, pertinência, auto-estima). Nessa perspectiva, a autora relata que o trabalho traz recompensas para os indivíduos com deficiência, fazendo com que muitos optem por um emprego ao invés de se manterem com os auxilios assistenciais. $\mathrm{O}$ trabalho possibilita uma relativa independência financeira, contribui para o auto-sustento e desperta a sensação de aceitação e de pertencimento numa população que, ao longo da história, sempre esteve às margens das oportunidades (Amaral, 1996).

Da mesma forma que o trabalho, a concepção de deficiência carrega uma evolução histórica nas várias sociedades (Amaral, 1996; Mendes, 1996). A deficiência pode significar descrédito social ou desvantagem para alguns, assim como inspirar pena em outros. Tratando-se da deficiência física, o estigma pode ser mais reforçado (Vash, 1988), já que esta implica visivelmente o comprometimento de alguma capacidade motora e a alteração da estrutura de um ou mais segmentos do corpo humano (Gargiulo, 2001; Hallahan \& Kauffman, 2003). Para avaliar a inserção de pessoas com deficiência física no mercado de trabalho, Galvani (2001) entrevistou 28 indivíduos com deficiência física, egressos de um programa de reabilitação profissional. As dificuldades encontradas por essas pessoas na busca de emprego referiram-se a obstáculos arquitetônicos (falta de adaptação nos transportes coletivos e nos mobiliários das empresas), a barreiras sociais (discriminação quanto à aparência física, preconceito, descrédito relacionado à capacidade, pouca oportunidade) e à baixa escolarização. No processo de ajustamento, Vash (1988) destaca a atratividade física e a assertividade como variáveis críticas em termos de recursos nos indivíduos com deficiência física.

A inclusão da pessoa com deficiência no mercado de trabalho é uma das finalidades e um dos objetos de investigação da área de Educação Especial. Contudo, a produção científica no Brasil sobre essa especificidade é escassa. Ao apresentar os núcleos temáticos do estudo da 
arte, na produção de conhecimento discente dos programas de pós-graduação stricto sensu, sobre indivíduos com necessidades educativas especiais das últimas décadas, Nunes, Ferreira, Mendes e Glat (2003) mostraram que os processos de integração/inclusão e profissionalização estão entre os menos freqüentes. No tema integração/inclusão, o local mais investigado foi a escola. A população-alvo mais freqüentemente estudada foi a de pessoas com deficiência mental. As pesquisas brasileiras na temática do trabalho são também desenvolvidas, em boa parte, com indivíduos especificamente com deficiência mental (Araújo, Escobal \& Goyos, 2006; Escobal, Araújo \& Goyos, 2005; Manzini, 1989; Ragazzi, 2001). Assim, parece ser relevante investigar os significados do trabalho para as pessoas com deficiência física inseridas em atividades laborais. Os resultados dessas pesquisas podem ser extremamente úteis para o estabelecimento de objetivos de ensino tanto na formação profissional quanto na orientação da própria organização empresarial, visando à efetiva inclusão dessa população.

Portanto, considerando: (a) a importância do trabalho para a identidade e o desenvolvimento das pessoas com deficiência; e (b) as dificuldades relacionadas à inserção no mercado de trabalho; (c) a necessidade de acompanhar a inclusão profissional de indivíduos com deficiência para verificar o ajustamento dessas pessoas ao emprego; (d) a escassez de conhecimento produzido sobre o acompanhamento profissional de pessoas com deficiência física, esta pesquisa teve por objetivos identificar e categorizar, no discurso dos participantes, aspectos positivos e negativos associados ao trabalho e comparar diferenças ou semelhanças desse discurso em relação aos trabalhadores com e sem deficiência física.

\section{Método}

\section{Participantes}

Formando dois grupos emparelhados em sexo, idade, estado civil, classe econômica e função, participaram da pesquisa: (a) 27 trabalhadores com deficiência física (TDF); e (b) 27 trabalhadores sem deficiência física (TND). O critério de inclusão na amostra foi o pareamento com outros participantes nas variáveis: sexo, idade e função. Ambos os grupos foram compostos por 14 homens (51,9\%) e 13 mulheres (48,1\%), com média de idade de 29 anos ( $d p=7,3$; $d p=7,9)$. Dentre os participantes, $75,9 \%$ possuíam níveis econômicos B2 e C, sendo o restante enquadrado nas classes B1 e A2, segundo o Critério de Classificação Econômica Brasil. A maioria dos participantes ocupava o cargo de auxiliar administrativo, possuía o ensino médio completo e era solteira. Grande parte das limitações físicas do grupo TDF foi devida à amputação de membros superiores e/ou inferiores, seguida de deformações congênitas, artrite reumatóide juvenil e escoliose grave. Quanto às demais variáveis emparelhadas, após a coleta de dados, a análise estatística não apontou diferença significativa entre os grupos TDF e TND.

\section{Local}

A realização do estudo ocorreu em cinco empresas de uma cidade do interior do estado de São Paulo com aproximadamente 600 mil habitantes. As atividades das empresas variaram entre comércio atacadista de produtos alimentícios e não-alimentícios, comércio varejista de medicamentos e de cosméticos, prestação de serviços médicos e hospitalares, prestação de serviços de manutenção do sistema viário, indústria e comércio de panificação.

\section{Instrumentos}

Critério de Classificação Econômica Brasil (www.abep.org). Com base no levantamento socioeconômico do Instituto Brasileiro de Opinião Pública e Estatística (IBGE), este instrumento é utilizado para identificar o nível econômico do participante. Por meio de questões fechadas, as respostas ao instrumento resultam em uma das seguintes classes: A1, A2, B1, B2, C, D ou E.

Questionário sobre Aspectos do Trabalho (QAT). A primeira parte caracteriza tempo de estudo, estado civil dos trabalhadores e aspectos relacionados a sua inserção no trabalho como, por exemplo, tempo na empresa e função. $\mathrm{Na}$ segunda parte, solicita-se que o participante fale sobre aspectos positivos e negativos associados ao trabalho. Não há nenhuma categoria prévia como guia de seu discurso. $\mathrm{O}$ trabalhador recebe a instrução para citar, pelo menos, três aspectos positivos e/ou três aspectos negativos.

\section{Procedimento de coleta de dados}

Realizou-se entrevista estruturada individual em aproximadamente 30 minutos, com base nos dois instrumentos. Após o consentimento dos participantes, estes foram instruídos quanto ao preenchimento do Critério de Classificação Econômica Brasil e, em seguida, do Questionário sobre Aspectos do Trabalho (QAT). A aplicação dos instrumentos foi presencial e semelhante em todas as empresas. A pesquisadora fez a leitura das questões e anotou as respostas dos participantes.

\section{Tratamento dos dados}

O resultado do Critério de Classificação Econômica Brasil foi obtido de acordo com a 
pontuação do próprio instrumento, computando-se posteriormente a sua freqüência. Com relação ao QAT, na análise dos aspectos associados ao trabalho, transcreveu-se o discurso dos participantes e, na seqüência, desenvolveu-se uma lista de registro dos temas, que foram então agrupados de acordo com o significado, recebendo uma denominação. Por exemplo, os temas agrupados nas subclasses trabalho repetitivo e alienação do trabalho foram depois reagrupados na classe mais geral denominada mecanização do trabalho. O agrupamento e a nomeação das classes e subclasses foram analisados por três juízes peritos na área, obtendo-se um índice de concordância de 85\%. Realizou-se, adicionalmente, análise de comparação entre os resultados dos grupos TDF e TND por meio do teste Qui-quadrado $(\chi 2)$ no SPSS 10.0 for Windows.

\section{Resultados}

A análise de conteúdo dos temas do questionário resultou, portanto, em classes de aspectos positivos e negativos associados ao trabalho, que foram então definidas. A relação e as respectivas definições das classes de aspectos positivos são explicitadas a seguir:

a) Cidadania. Diz respeito ao pleno exercício dos direitos e deveres proporcionados pela sociedade e pela legislação, principalmente na questão da saúde, da segurança, da dignidade e do respeito.

b) Conciliação de interesses. Refere-se à possibilidade de negociação com relação à flexibilização do horário de trabalho, facilitando a garantia de aproveitamento do tempo em interesses alheios ao tempo de expediente.

c) Finanças. Garantia à estabilidade e à disponibilidade financeira para a satisfação das necessidades básicas de sobrevivência.

d) Relações sociais. Refere-se à condição favorável de relacionamento interpessoal satisfatório no contexto do trabalho que facilite o convívio e o desenvolvimento social dos trabalhadores.

e) Valorização pessoal. Condições que visam ao desenvolvimento cognitivo e emocional favorecidos pelas diversas experiências no cotidiano do trabalho, possibilitando satisfação pessoal.

f) Valorização profissional. Indicativo de possibilidade de desenvolvimento da capacidade para o trabalho de forma a proporcionar oportunidades e reconhecimento profissional.

A Figura 1 mostra a freqüência de respostas nas classes de aspectos positivos para o grupo TDF e para o grupo TND.

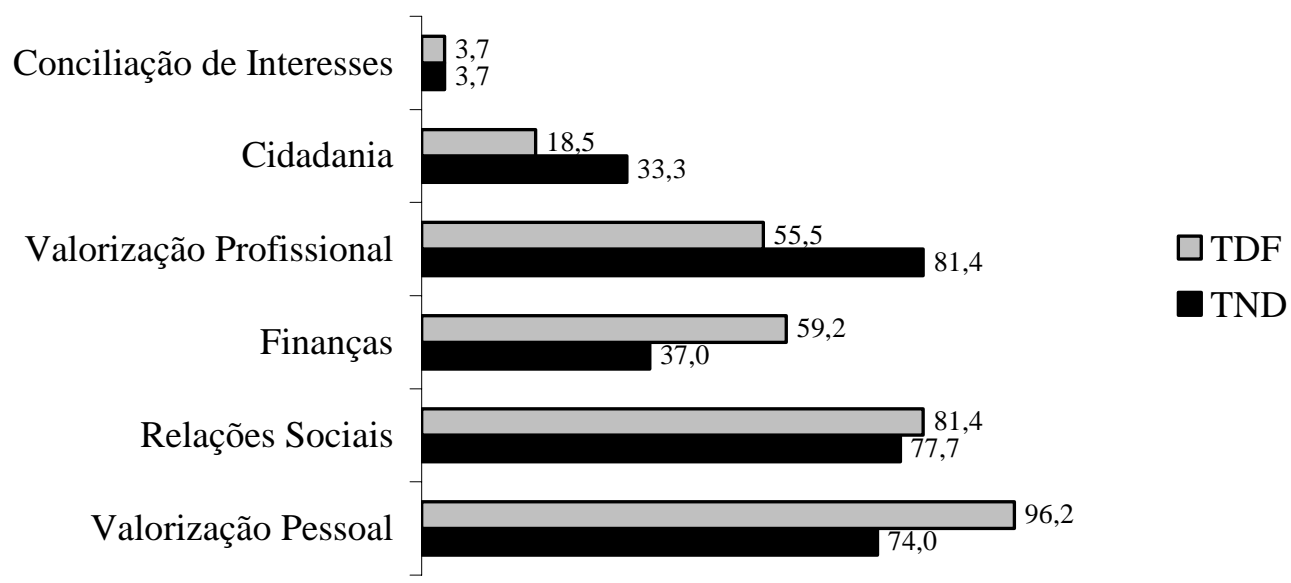

Figura 1 - Freqüência relativa (\%) das classes de aspectos positivos associados ao trabalho entre os participantes dos grupos TDF e TND

Observa-se na Figura 1 que, enquanto valorização pessoal e, na seqüência, relações sociais foram os aspectos positivos do trabalho mais freqüentemente referidos pelo grupo TDF, o grupo TND indicou valorização profissional e, posteriormente, relações sociais. É interessante notar que, tanto para o grupo TDF quanto para o grupo
TND, a classe finanças apresentou maior freqüência do que cidadania. Chama a atenção, ainda, que a diferença de indicação entre essas duas classes foi muito maior no grupo TDF.

$\mathrm{Na}$ Tabela 1, encontram-se os resultados da comparação das classes de aspectos positivos associados ao trabalho entre os grupos TDF e TND. 
Tabela 1 - Freqüência e comparação de aspectos positivos do trabalho entre TDF e TND

\begin{tabular}{|c|c|c|c|}
\hline Classes & Subclasses & TDF & TND \\
\hline Valorização & Ser útil & 22,2 & 7,4 \\
\hline \multirow{15}{*}{ pessoal } & Auto-estima & 7,4 & - \\
\hline & Autocontrole & 3,7 & 3,7 \\
\hline & Realce à alegria & 3,7 & - \\
\hline & Otimismo & - & 3,7 \\
\hline & Confirmação da capacidade para o trabalho & 7,4 & - \\
\hline & Autonomia & 22,2 & 7,4 \\
\hline & Responsabilidade & 7,4 & 14,8 \\
\hline & Superação de obstáculos & 3,7 & - \\
\hline & Estímulo à perseverança & 3,7 & - \\
\hline & Satisfação pessoal & - & 3,7 \\
\hline & Fortalecimento do caráter & - & 3,7 \\
\hline & Autovalorização & - & 3,7 \\
\hline & Desenvolvimento pessoal & 11,1 & 18,5 \\
\hline & Desafios & 3,7 & - \\
\hline & Disciplina & - & 7,4 \\
\hline \multirow[t]{10}{*}{ Relações sociais } & Perda da timidez & 18,5 & 29,6 \\
\hline & Relacionamento com outras pessoas & 29,6 & 22,2 \\
\hline & Desenvolvimento da assertividade & 3,7 & - \\
\hline & Desenvolvimento social & - & 7,4 \\
\hline & Bom relacionamento com colegas de trabalho & 3,7 & - \\
\hline & Socialização & 7,4 & 11,1 \\
\hline & Cooperação & 3,7 & - \\
\hline & Amizades & 11,1 & - \\
\hline & Trabalho em grupo & - & 7,4 \\
\hline & Trabalhar com pessoas humoradas & 3,7 & - \\
\hline Valorização & Aprendizagem & 25,9 & 25,9 \\
\hline \multirow[t]{8}{*}{ profissional } & Aprimoramento das habilidades técnicas & - & 3,7 \\
\hline & Crescimento profissional & 25,9 & 29,6 \\
\hline & Resolução de problemas & - & 3,7 \\
\hline & Tomada de decisão & - & 3,7 \\
\hline & Desenvolvimento intelectual & - & 7,4 \\
\hline & Novas oportunidades profissionais & - & 3,7 \\
\hline & Reconhecimento profissional & - & 3,7 \\
\hline & Satisfação profissional & 3,7 & - \\
\hline \multirow[t]{7}{*}{ Cidadania } & Benefícios assistenciais e previdenciários & 3,7 & 7,4 \\
\hline & Fazer parte da sociedade & - & 3,7 \\
\hline & Bem-estar da família & - & 3,7 \\
\hline & Segurança & - & 3,7 \\
\hline & Ser integrante do mundo do trabalho & 11,1 & - \\
\hline & Dignidade & 3,7 & 7,4 \\
\hline & Respeito & - & 3,7 \\
\hline \multirow[t]{3}{*}{ Finanças } & Estabilidade financeira & 40,7 & 14,8 \\
\hline & Auto-sustento & 11,1 & 22,2 \\
\hline & Dispor de receita própria para o lazer & 7,4 & - \\
\hline $\begin{array}{l}\text { Conciliação de } \\
\text { interesses }\end{array}$ & Horário que possibilita fazer outras coisas & 3,7 & 3,7 \\
\hline
\end{tabular}

Nota-se que as classes compostas por maior diversidade de subclasses foram respectivamente: valorização pessoal (16 subclasses); relações sociais (10 subclasses); valorização profissional (nove subclasses); 
cidadania (sete subclasses); finanças (três subclasses); e conciliação de interesses (uma subclasse). A análise estatística $(\chi 2)$ não mostrou diferença significativa entre TDF e TND na freqüência das classes gerais de aspectos positivos associados ao trabalho.

As classes de aspectos negativos do trabalho identificadas neste estudo foram:

a) Desgaste físico e emocional. Indica excesso de carga de trabalho, provocando o cansaço, a preocupação e o estresse.

b) Desvalorização profissional. Política organizacional caracterizada por baixas perspectivas de crescimento, falta de reconhecimento, remuneração inadequada geradora de insatisfação e estagnação no trabalho. c) Dificuldades interpessoais. Refere-se à competitividade geradora de conflitos interpessoais resultantes das dificuldades em conviver com diferentes pessoas no contexto do trabalho.

d) Jornada de trabalho extensa. Refere-se ao expediente abusivo de trabalho e pouco flexível, resultando em dificuldades para conciliar as demandas pessoais e familiares dos empregados.

e) Mecanização do trabalho. Condição de execução repetitiva com prejuízo da criatividade e facilitação da alienação do pensamento crítico dos empregados.

A Figura 2 apresenta a freqüência de ocorrência das classes de aspectos negativos associados ao trabalho entre os grupos TDF e TND.

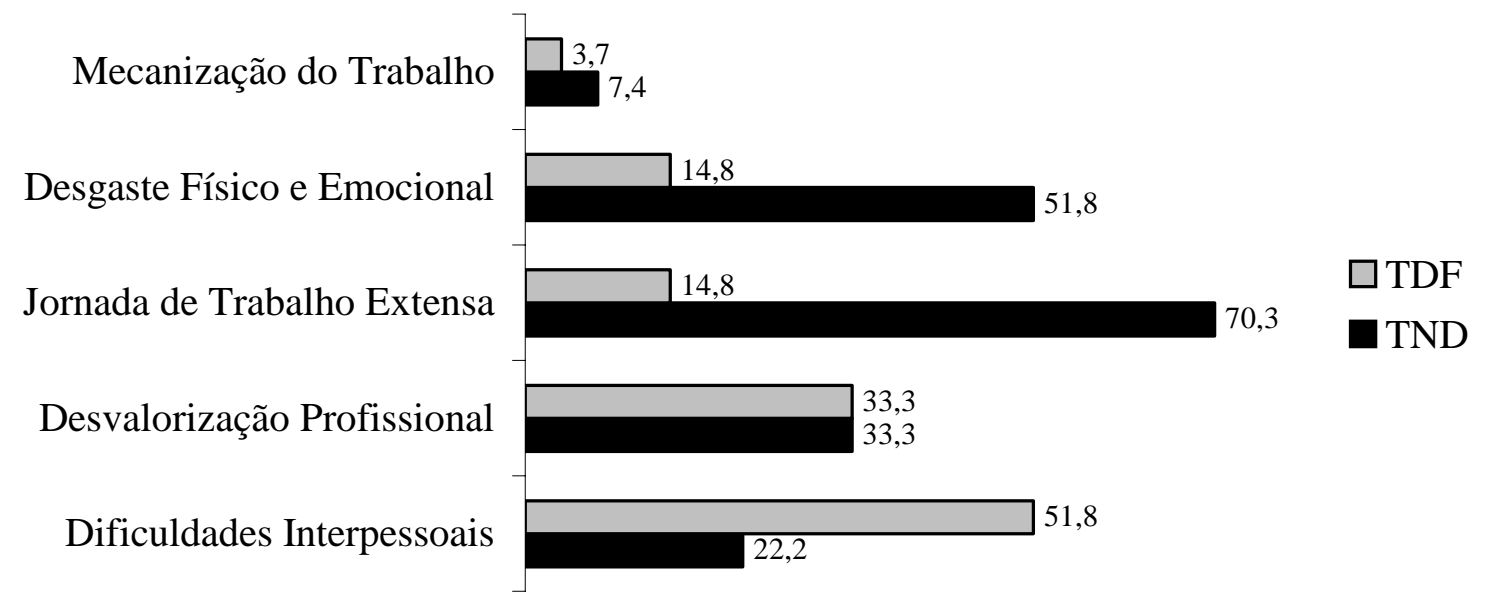

Figura 2 - Freqüência relativa (\%) das classes de aspectos negativos associados ao trabalho entre os participantes dos grupos TDF e TND

Como se observa na Figura 2, em relação aos aspectos negativos associados ao trabalho, o grupo TDF indicou maior freqüência relativa para dificuldades interpessoais e desvalorização profissional do que para as demais classes identificadas. Enquanto isso, TND apontou jornada de trabalho extensa e desgaste físico e emocional.

A Tabela 2 compara as classes de aspectos negativos associados ao trabalho entre TDF e TND, revelando suas subclasses.
As classes compostas por maior diversidade de subclasses foram: dificuldades interpessoais (nove subclasses); jornada de trabalho extensa (oito subclasses); desvalorização profissional (seis subclasses); desgaste físico e emocional (seis subclasses); e mecanização do trabalho (duas subclasses). A análise comparativa $(\chi 2)$ indicou diferença entre os grupos na classe jornada de trabalho extensa, sendo que a freqüência foi significativamente mais elevada para o grupo TND do que para o grupo TDF $\left(\chi 2_{(1)}=8,333 ; p<0,01\right)$. 
Tabela 2 - Freqüência e comparação de aspectos negativos do trabalho entre TDF e TND

\begin{tabular}{|c|c|c|c|}
\hline Classes & Subclasses & TDF & TND \\
\hline \multirow{9}{*}{$\begin{array}{l}\text { Dificuldades } \\
\text { interpessoais }\end{array}$} & Conflitos interpessoais & 14,8 & 3,7 \\
\hline & Convívio com pessoas arrogantes & 3,7 & - \\
\hline & Trabalhar com pessoas mal-humoradas & 3,7 & - \\
\hline & Dificuldade de trabalhar em grupo & 3,7 & 3,7 \\
\hline & Dificuldade de lidar com críticas & 7,4 & - \\
\hline & Competição excessiva e desleal & - & 11,1 \\
\hline & Autoritarismo de colegas de trabalho & 3,7 & - \\
\hline & Desrespeito & 14,8 & - \\
\hline & Falta de ética & - & 3,7 \\
\hline \multirow{7}{*}{$\begin{array}{l}\text { Jornada de } \\
\text { trabalho extensa }\end{array}$} & Pouco tempo disponível para a família & - & 18,5 \\
\hline & Redução de tempo para os estudos & 3,7 & 11,1 \\
\hline & Pouco tempo para o lazer & - & 14,8 \\
\hline & Trabalhar no fim de semana & 3,7 & 7,4 \\
\hline & Constantes mudanças de horário & - & 7,4 \\
\hline & Turno de trabalho noturno & 3,7 & - \\
\hline & Horário incompatível com compromissos pessoais & 3,7 & 11,1 \\
\hline \multirow{6}{*}{$\begin{array}{c}\text { Desvalorização } \\
\text { profissional }\end{array}$} & Remuneração inadequada & 11,1 & 14,8 \\
\hline & Baixa perspectiva de crescimento & - & 3,7 \\
\hline & Insatisfação profissional & - & 3,7 \\
\hline & Falta de reconhecimento & 18,5 & 3,7 \\
\hline & Estagnação & - & 3,7 \\
\hline & Posto de trabalho inadequado & 3,7 & 3,7 \\
\hline \multirow{6}{*}{$\begin{array}{l}\text { Desgaste físico } \\
\text { e emocional }\end{array}$} & Estresse & 7,4 & 25,9 \\
\hline & Preocupação & - & 3,7 \\
\hline & Cansaço & - & 11,1 \\
\hline & Ambiente tenso & - & 3,7 \\
\hline & Responsabilidade excessiva & 7,4 & 3,7 \\
\hline & Cobrança excessiva & - & 3,7 \\
\hline \multirow{2}{*}{$\begin{array}{c}\text { Mecanização do } \\
\text { trabalho }\end{array}$} & Trabalho repetitivo & 3,7 & 3,7 \\
\hline & Alienação & - & 3,7 \\
\hline
\end{tabular}

\section{Discussão}

Em relação às classes de aspectos positivos associados ao trabalho, embora a análise estatística não tenha indicado nenhuma diferença significativa entre os grupos TDF (trabalhadores com deficiência física) e TND (trabalhadores sem deficiência física), a freqüência relativa das classes de respostas que emergiram de cada grupo, permite algumas ilações. Para o grupo TDF, valorização pessoal foi uma classe na qual os participantes apresentaram maior número de respostas, enquanto para o grupo TND foi valorização profissional. Esse resultado apóia as considerações dadas por estudiosos da área de Educação Especial (Amaral, 1994; Anache, 1996; Chadsey \& Beyer, 2001; Dalferth, Schnappauf \& Sommerer, 1995; Ragazzi, 2001; Vash, 1988), que enfatizaram as diversas recompensas ocasionadas pelo trabalho e sua importância para o desenvolvimento socioemocional dos indivíduos com deficiência.
Quanto aos aspectos negativos associados ao trabalho, apenas a classe jornada de trabalho extensa apresentou diferença significativa, superior para o grupo TND em relação ao grupo TDF. Tal achado sugere a ocorrência de mais abusos sobre esse grupo em relação ao expediente de trabalho, com pouco tempo restante para as outras necessidades pessoais, do que para os participantes com deficiência física. Vários fatores podem estar envolvidos nessa diferenciação. $\mathrm{O}$ grupo TDF pode: (a) não se achar com direito de fazer exigências por se considerar inferiorizado em relação aos demais; (b) considerar a oportunidade de trabalho mais como uma concessão do que propriamente um contrato legal de compra e venda da força do trabalho regido pela legislação; (c) sobrevalorizar os ganhos indiretos do trabalho, como a oportunidade de fazer novos amigos e se relacionar com as pessoas. Quanto ao grupo TND, é possível supor que os participantes: (a) têm maior conhecimento sobre seus direitos e compreendem $\mathrm{O}$ 
trabalho dentro da perspectiva da legislação; e (b) sentemse mais capazes para obter outro emprego caso sejam desligados da empresa.

$\mathrm{Na}$ investigação da classe de aspecto negativo do trabalho mais freqüentemente citada pelo grupo TDF em comparação com o grupo TND, encontra-se exclusivamente dificuldades interpessoais. Esse resultado é concordante com dados de outras pesquisas que revelaram problemas interpessoais em torno de pessoas com deficiência física por diferentes explicações: déficits de habilidades sociais no repertório comportamental do próprio indivíduo (Pereira, Del Prette \& Del Prette, sd; Thomas, Bax \& Smyth, 1988; Vash, 1988), autoconceito insatisfatório (Hallahan \& Kauffman, 2003), despreparo da sociedade para lidar com as diferenças (Amaral, 1994; Carreira, 1997; Galvani, 2001), ineficiência da profissionalização nesse quesito (Del Prette \& Del Prette, 2003; Tanaka \& Manzini, 2005).

Por fim, na classe desvalorização profissional, destaca-se a freqüência de respostas indicada pelos participantes do grupo TDF para a subclasse falta de reconhecimento. Anache (1996), Galvani (2001) e Lancillotti (2003) identificaram, em seus estudos, que a maioria de trabalhadores com deficiência desempenhava funções operacionais e raramente ocupava uma função de chefia nas empresas, o que pode sugerir vários motivos, entre os quais: (a) falta de investimento na capacitação funcional da organização nesse estrato; (b) descrença quanto à capacidade da pessoa com deficiência em aprender e liderar; (c) acomodação dos próprios indivíduos com deficiência na função.

\section{Considerações finais}

Os resultados desta pesquisa permitiram identificar diferentes classes de aspectos positivos e negativos associados ao trabalho na perspectiva de trabalhadores com e sem deficiência física. Conclui-se que a deficiência física não foi um fator determinante na valorização de aspectos positivos e negativos do trabalho. Todavia, como os trabalhadores com deficiência física possuem necessidades especiais, reconhecer o significado que atribuem ao trabalho remete a aspectos pertinentes ao ajustamento deles no contexto profissional.

É possível perceber que, embora o trabalho possa conferir valor social e pessoal ao empregado com deficiência física, são necessárias providências efetivas no âmbito laboral para que ocorra a inclusão destes nesse ambiente e na sociedade. Destaca-se, neste estudo, a necessidade de programas de treinamento (na educação profissional/superior e nas empresas) que ultrapassem os itens de desempenhos funcionais e incluam aspectos próprios dos relacionamentos interpessoais no contexto do trabalho (Del Prette \& Del Prette, 2003; Rodrigues, Rubio \& Expósito, 1995), principalmente quando se depara com o fato de que indivíduos com deficiência são mais vulneráveis a perderem seus empregos em virtude de dificuldades nas habilidades sociais (Antonello, 1996).

Os achados da presente pesquisa apontam para a relevância da preparação do trabalhador nas relações interpessoais, tanto do grupo TDF como do grupo TND, considerando a inclusão como um processo que ocorre nas interações entre as pessoas. Adicionalmente, a investigação sobre aspectos positivos e negativos do trabalho pode contribuir para uma prática mais efetiva do setor de gestão de pessoas, bem como na implementação, por parte das empresas, de programas de ações afirmativas, visando promover a igualdade de oportunidades e direitos. Concomitantemente a essa questão, encontra-se a importância das instituições profissionalizantes (escolas e universidades) reavaliarem se suas ações estão sendo condizentes com as demandas do mercado de trabalho.

Não obstante tais considerações, novas pesquisas com amostras ampliadas em diferentes pontos do país poderiam trazer dados importantes para subsidiar políticas públicas sobre a inserção no mercado de trabalho dos indivíduos com necessidades educativas especiais.

\section{Referências}

Amaral, L. A. (1994). Mercado de trabalho e deficiência. Revista Brasileira de Educaşão Especial, 1, 127-136.

Amaral, L. A. (1996). Algumas reflexões sobre a (r)evolução do conceito da deficiência. Em C. Goyos, M. A. Almeida \& D. G. Souza (Orgs.). Temas em educação especial (Vol. 3, pp. 99-106). São Carlos: EDUFSCar.

Anache, A. A. (1996). O deficiente e o mercado de trabalho: concessão ou conquista? Revista Brasileira de Educação Especial, 2, 119-126.

Antonello, S. J. (1996). Social skills development: Practical strategies for adolescents and adults with developmental disabilities (pp. 210-232). Boston: Allyn and Bacon.

Antunes, R. (2003). Os caminhos da liofilização organizacional: as formas diferenciadas $\mathrm{da}$ reestruturação produtiva no Brasil. Idéias, 10, 13-24.

Araújo, E. A. C., Escobal, G. \& Goyos, C. (2006). Programa de suporte comunitário: alternativa para $\mathrm{O}$ trabalho do adulto deficiente mental. Revista Brasileira de Educação Especial, 12, 221-240.

Borges, L. O. \& Alves-Filho, A. (2001). A mensuração da motivação e do significado do trabalho. Estudos de Psicologia, 6, 177-194. 
Borges, L. O. \& Yamamoto, O. H. (2004). O mundo do trabalho. Em J. C. Zanelli, J. E. Borges-Andrade \& A. V. B. Bastos (Orgs.). Psicologia, organizações e trabalho no Brasil (pp. 24-62). São Paulo: Artmed.

Bottomore, T. (1988). Dicionário do pensamento marxista. Rio de Janeiro: Jorge Zahar.

Braverman, H. (1987). Trabalho e capital monopolista: a degradação do trabalho no século XX. São Paulo: Guanabara.

Carreira, D. (1997). A integração da pessoa deficiente no mercado de trabalho. Em M. T. E. Mantoan (Org.). A integração de pessoas com deficiência: contribuições para uma reflexão sobre o tema (pp. 24-31). São Paulo: Memnon.

Chadsey, J. \& Beyer, S. (2001). Social relationships in the workplace. Mental Retardation and Developmental, 7, 128-133.

Chahad, J. P. Z. (2003). Tendências recentes no mercado de trabalho: pesquisa de emprego e desemprego. São Paulo em Perspectiva, 17, 205-217.

Dalferth, M., Schnappauf, K. \& Sommerer, L. (1995). Vocational integration of physically handicapped persons: A regional study in the Regensburg district. Rehabilitation, 34, 91-100.

Dejours, C., Abdoucheli, E. \& Jayet, C. (1994). Psicodinâmica do trabalho: contribuições da escola dejouriana à análise da relação pražer, sofrimento e trabalho. São Paulo: Atlas.

Del Prette, Z. A. P \& Del Prette, A. (2003). Desenvolvimento interpessoal: uma questão pendente no ensino universitário. Em E. Mercuri \& S. A. J. Polydoro (Orgs.). Estudante universitário: características e experiências de formação (pp. 105-128). Taubaté: Cabral.

Escobal, G., Araújo, E. A. C. \& Goyos, C. (2005). Escolha e desempenho no trabalho de adultos com deficiência mental. Revista Brasileira de Educação Especial, 11, 355-372.

Forrester, V. (1997). O horror econômico. São Paulo: UNESP.

Galvani, R. C. D. (2001). Inserção do deficiente físico no mercado de trabalho: a ótica da instituição especializada e a ótica do egresso Dissertação de Mestrado. Marília: Universidade Estadual Paulista - Pós-Graduação em Educação.

Gargiulo, R. M. (2001). Special education in contemporary society: An introduction to exceptionality (pp. 533-547). Nova Iorque: Wadsworth.
Glina, D. M. R. \& Rocha, L. E. (2003). Exigências do trabalho, prevalência de dor muscular e sintomas de estresse em estagiários do setor de cobrança de um banco internacional. Revista de Terapia Ocupacional da Universidade de São Paulo, 14, 10-18.

Hallahan, D. P. \& Kauffman, J. M. (2003). Exceptional leaners: Introduction to special education (pp. 431-451). Nova Iorque: Allyn and Bacon.

Jaime, L. R. \& Carmo, J. C. (2005). A inserção da pessoa com deficiência no mundo do trabalho: o resgate de um direito de cidadania. São Paulo: Ed. dos Autores.

Kovács, I. (2004). Emprego flexível em Portugal. Sociologias, 12, 32-67.

Lancillotti, S. S. P. (2003). Deficiência e trabalho: redimensionando o singular no contexto universal. Campinas: Autores Associados.

Manzini, E. J. (1989). Profissionalização de individuos deficientes mentais: visão do agente institucional e visão do egresso. Dissertação de Mestrado. São Carlos: Universidade Federal de São Carlos - Pós-Graduação em Educação Especial.

Mendes, A. M., Borges, L. O. \& Ferreira, M. C. (Orgs.) (2002). Trabalho em transição, saúde em risco. Brasília: $\mathrm{UnB}$.

Mendes, E. G. (1996). Evolução histórica da concepção científica de deficiência mental. Em C. Goyos, M.A. Almeida \& D. G. Souza (Orgs.). Temas em educação especial (Vol. 3, pp. 119-136). São Carlos: EDUFSCar.

Nunes, L. R. P., Ferreira, J. R., Mendes, E. G. \& Glat, R. (2003). Análise das dissertações e teses sobre educação especial nas áreas de educação e psicologia. Em M. C. Marquezine, M. A. Almeida \& S. Omote (Orgs.). Colóquios sobre pesquisa em educação especial (pp. 65-73). Londrina: EDUEL.

Pereira, C. S, Del Prette, A. \& Del Prette, Z. A. P. (sd). Habilidades sociais entre trabalhadores com e sem deficiência física: um estudo comparativo. The Spanish Journal of Psychology.

Ragazzi, C. L. M. (2001). Emprego com apoio: alternativa viável para inserção de pessoas com deficiência mental no mercado de trabalho? Dissertação de Mestrado. São Carlos: Universidade Federal de São Carlos - PósGraduação em Educação Especial.

Rodrígues, F. G., Rubio, J. M. L. \& Expósito, L. J. (1995). Habilidades sociales y salud (pp. 139-146). Madri: Eudema.

Sarriera, J. C., Câmara, S. G. \& Berlim, C. S. (2000). Elaboração, desenvolvimento e avaliação de um programa de inserção ocupacional para jovens 
desempregados. Psicologia: Reflexão e Crítica, 13, 189198.

Sarriera, J. C., Silva, M. A., Kabbas, C. P. \& Lopes, V. B. (2001). Formação da identidade ocupacional em adolescentes. Estudos em Psicologia, 6, 27-32.

Tanaka, E. D. O. \& Manzini, E. J. (2005). O que os empregadores pensam sobre o trabalho da pessoa com deficiência? Revista Brasileira de Educação Especial, 11, 273-294.

Thomas, A. P., Bax, M. C. \& Smyth, D. P. (1988). The social skill difficulties of young adults with physical disabilities. Child: Care, Health and Development, 14, 255-264.

Vash, C. L. (1988). Enfrentando a deficiência: a manifestação, a psicologia, a reabilitação (pp. 101-123). São Paulo: Pioneira.

Sobre os autores:

Camila de Sousa Pereira é psicóloga, doutoranda pelo Programa de Pós-Graduação em Educação Especial da Universidade Federal de São Carlos (UFSCar), São Carlos-SP.

Almir Del Prette é professor titular do Departamento de Psicologia e do Programa de Pós-Graduação em Educação Especial da Universidade Federal de São Carlos (UFSCar), São Carlos-SP.

Zilda Aparecida Pereira Del Prette é professora titular do Departamento de Psicologia e do Programa de PósGraduação em Educação Especial da Universidade Federal de São Carlos (UFSCar), São Carlos-SP. 